\title{
Hydra effects in stable communities and their implications for system dynamics
}

\author{
Michael H. Cortez,,$^{1,3}$ and Peter A. Abrams² \\ ${ }^{1}$ Department of Mathematics and Statistics, Utah State University, Logan, Utah 84322, USA \\ ${ }^{2}$ Department of Ecology and Evolutionary Biology, University of Toronto, 25 Harbord St., Toronto, ON M5S 3G5, Canada
}

\begin{abstract}
A hydra effect occurs when the mean density of a species increases in response to greater mortality. We show that, in a stable multispecies system, a species exhibits a hydra effect only if maintaining that species at its equilibrium density destabilizes the system. The stability of the original system is due to the responses of the hydra-effect species to changes in the other species' densities. If that dynamical feedback is removed by fixing the density of the hydra-effect species, large changes in the community make-up (including the possibility of species extinction) can occur. This general result has several implications: (1) Hydra effects occur in a much wider variety of species and interaction webs than has previously been described, and may occur for multiple species, even in small webs; (2) conditions for hydra effects caused by predators (or diseases) often differ from those caused by other mortality factors; (3) introducing a specialist or a switching predator of a hydra-effect species often causes large changes in the community, which frequently involve extinction of other species; (4) harvest policies that attempt to maintain a constant density of a hydra-effect species may be difficult to implement, and, if successful, are likely to cause large changes in the densities of other species; and (5) trophic cascades and other indirect effects caused by predators of hydra-effect species can exhibit amplification of effects or unexpected directions of change. Although we concentrate on systems that are originally stable and models with no stage-structure or trait variation, the generality of our result suggests that similar responses to mortality will occur in many systems without these simplifying assumptions. In addition, while hydra effects are defined as responses to altered mortality, they also imply counterintuitive responses to changes in immigration and other parameters affecting population growth.
\end{abstract}

Key words: consumer-resource system; indirect effect; overcompensation; predator-prey; trophic cascade.

\section{INTRODUCTION}

Intuition suggests that increases in the mortality rate of a species, due to predation, disease, harsh environments or other factors, will decrease its population size. This assumption underlies many conservation and management strategies. However, increased mortality of a species can have the counterintuitive effect of increasing its population size. An increase in mean population size in response to higher mortality was first described by Ricker (1954), using a discrete model of a single homogeneous population that exhibited sustained fluctuations. Increased population size caused by increased mortality is now known as a "hydra effect" (Abrams and Matsuda 2005, Abrams 2009b). It has been described for several discrete models of single species growth and a variety of continuous time models of simple food webs. See Abrams (2009b) for a review and Abrams (2009a, 2012), Sieber and Hilker (2012), Guill et al. (2014), Georgelin and Loeuille (2014), Sieber et al. (2014) and

Manuscript received 9 April 2015; revised 8 December 2015; accepted 15 December 2015. Corresponding Editor: S. J. Schreiber.

${ }^{3}$ E-mail: michael.cortez@usu.edu
Abrams and Cortez (2015) for a variety of recent examples. Hydra effects have been observed in empirical studies of ciliates (Fryxell et al. 2005), blowflies (Nicholson 1954, 1957), and fish (Zipkin et al. 2008, Schröder et al. 2009).

Despite this growing body of work, no unifying characteristic that predicts when a species will exhibit a hydra effect has been identified to date. Here we describe such a property for stable systems. Specifically, building upon the work of Yodzis (1988), we derive a general condition that determines when species exhibit hydra effects in differential equation models of homogeneous populations with stable equilibrium points. We find that a species exhibits a hydra effect only if the subcommunity 'composed of' the other species is inherently unstable. By this we mean that if the density of the species with the hydra effect is maintained at its equilibrium value, then large community shifts occur; these shifts include the possibility of extinction of one or more species. This result is surprising because fixing the density of the hydra-effect (HE) species does not change its density. Instead, fixing the density removes the ability of the HE species to respond to changes in the densities of the other species, 
and consequently, the dynamical feedback of the HE species on the community; this feedback is necessary for system stability. Hence, community stability requires the ability of the HE species to vary with changes in the densities of other species. We refer to the scenario where a system is unstable when the density of an HE species is fixed as "subsystem instability."

Using our general result, we derive the conditions under which hydra effects arise in a suite of two- and threespecies ecological modules without stage structure. This reveals the existence of hydra effects in many simple communities where they have not previously been described. The subsystem instability criterion implies that introducing a specialist predator that feeds on an HE species causes large changes in community composition, that frequently involve the extinction of one or more non-HE species. The subsystem instability criterion also implies that trophic cascades and other indirect effects due to the introduced specialist predator frequently display amplification, meaning that the indirect effects of the introduction are much larger than its direct effect on the HE species. Other aspects of indirect effects that pass through an HE species differ from those predicted for non-hydra species. We discuss how the subsystem instability result can be used to uncover when hydra effects arise in a broader class of models having adaptive dynamics of traits and/or stage-structure. Finally, we discuss how our result implies the potential for unexpected impacts of human management of species that exhibit hydra effects.

\section{$N$-SPECIES COMMUNITY MODEL AND METHODS}

The general $n$-species community model describes how the density of each species $\left(x_{j}, 1 \leq j \leq n\right)$ changes over time,

$$
\frac{\mathrm{d} x_{j}}{\mathrm{~d} t}=x_{j} f_{j}\left(x_{1}, \ldots, x_{n}\right)-\delta_{j} x_{j}
$$

where $\delta_{j}$ is the density-independent component of the per capita mortality rate of species $j$ and the per capita growth rate function $f$ incorporates changes in the demographic rates of species $j$ due to all other ecological processes. The coexistence equilibrium of model (1) is denoted by $p=\left(x_{1}^{*}, \ldots, x_{n}^{*}\right)$. We assume the coexistence equilibrium is stable, i.e., all of the eigenvalues of the Jacobian $(J)$ evaluated at $p$ have negative real parts.

Given a stable equilibrium, species $i$ exhibits a hydra effect when $\partial x_{i}^{*} / \partial \delta_{i}(p)>0$, i.e., the equilibrium density of species $i$ increases with increased density-independent mortality. Yodzis (1988) showed that the response to increased immigration in species $i$ is $-\left(J^{-1}\right)_{i i}$, where $J^{-1}$ is the inverse of the Jacobian evaluated at $p$. In our model, the effect of increased mortality of species $i$ on its equilibrium density has the opposite sign, $\partial x_{i}^{*} / \partial \delta_{i}=x_{i}^{*}\left(J^{-1}\right)_{i i}$. Substituting for the $i i$ element of the inverse Jacobian yields the following mathematical condition for a hydra effect,

$$
\frac{\partial x_{i}^{*}}{\partial \delta_{i}}=\frac{x_{i}^{*} M_{i i}}{\operatorname{det}(J)}>0 ;
$$

see Appendix S1 for details. The term $M_{i i}$ is the $i$ th principle minor of the Jacobian, i.e., the determinant of the matrix from which the $i$ th row and $i$ th column of the Jacobian have been removed. For stable systems, species $i$ has a hydra effect if and only if $\operatorname{det}(J)$ and $M_{i i}$ have the same sign.

The signs of $\operatorname{det}(J)$ and $M_{i i}$ have important interpretations. For an even number of species ( $n$ even), stable coexistence implies det $(J)>0$. For an odd number of species ( $n$ odd), stable coexistence implies $\operatorname{det}(J)<0$. The sign of $M_{i i}$ has a similar interpretation for the system where the density of species $i$ is fixed at its equilibrium density $\left(x_{i}=x_{i}^{*}\right)$. The dynamics of this new system are governed by Eq. 1 after removing the $\mathrm{d} x_{i} / \mathrm{d} t$ equation and treating the density of species $i\left(x_{i}\right)$ as a controlled parameter. For a system with an even number of species ( $n$ even), stable coexistence of all species when the density of $x_{i}$ is fixed is possible only if $M_{i i}<0$. For an odd number of species ( $n$ odd), stable coexistence of all species when the density of $x_{i}$ is fixed is possible only if $M_{i i}>0$.

Equation 2 has three important implications; see Appendix S1 for details. First, this study focuses on hydra effects that arise via increased density-independent mortality $\left(\delta_{j}\right)$, but increases in density-dependent mortality have the same qualitative effect. Second, as noted by Yodzis (1988), the sign of the effect of decreased (increased) immigration of a species on equilibrium population size is the same as that produced by increased (decreased) mortality. Hence, if density-independent immigration were added to model (1), the effect of decreased immigration on equilibrium population size would be the right hand side of equation (2) divided by $x_{i}^{*}$. Third, and more generally, any parameter that affects a species' population growth but does not directly affect the dynamics of other species in the system, will have a counterintuitive effect on the affected species' population density under the same circumstances as increased mortality. Thus, e.g., decreasing a predator's conversion efficiency causes increased predator abundance under the same conditions for which hydra effects occur.

\section{HYDRA EFFECTS IMPLY SUBSYSTEM INSTABILITY}

Equation 2 means that in a stable system, species $i$ has a hydra effect if $\operatorname{det}(J)$ and $M_{i i}$ have the same sign. This implies that for a stable coexistence equilibrium, fixing the density of an HE species at its equilibrium value will destabilize the system. In many cases, this results in the extinction of one or more species. This result is surprising because the density of the species with the hydra effect has not changed. What has changed is the ability of the HE species to respond to changes in the densities of the other species. If the density of the HE species is not fixed, then small changes in the densities of the other species cause small changes in the HE species, which then drive the system back to the original equilibrium state. In contrast, when the density of the HE species is fixed, small changes in the densities of the other species grow. Hence, hydra 
effects arise in a species when the subset of the community that lacks dynamics of that species is inherently unstable. As noted in the Introduction, we refer to this as "subsystem instability". In a sense, the HE species represents a "keystone" species for stable coexistence of the remainder of the community. However, it is not just the presence of the HE species, but its ability to vary with changes in the densities of other species, that is required for stability.

Two qualifications to our result should be noted. First, hydra effects only occur over a limited range of mortality rates. Increasing the mortality rate of an $\mathrm{HE}$ species to sufficiently high levels will eventually cause its equilibrium density to either decrease with further increase in mortality or discontinuously drop to zero. Second, the subsystem instability result only necessarily applies when the density of the HE species $\left(x_{i}\right)$ is held at its equilibrium value $\left(x_{i}=x_{i}^{*}\right)$. However, for Lotka-Volterra models, where the functions $f_{j}$ are linear, the instability of the system where $x_{i}$ is fixed is independent of the value of $x_{i}$, provided no species go extinct as the density of that species is changed; see Appendix S1 for details. Because of this, in Lotka-Volterra systems, if species $i$ exhibits a hydra effect, then the other $n-1$ species cannot coexist at a stable equilibrium in the absence of that species. When the functions $f_{j}$ are sufficiently nonlinear, the destabilization result need not apply when the density of the HE species is held at some value sufficiently far from the original equilibrium. Systems with nonlinear functions may also have multiple coexistence equilibria, one or more of which is stable. Both of these cases may allow the $n-1$ species to coexist when the HE species is absent $\left(x_{i}=0\right)$ or fixed at a density different from its equilibrium density $\left(x_{i} \neq x_{i}^{*}\right)$.

We note two other general results linking hydra effects and subsystem instability. First, multiple species exhibit hydra effects whenever there are multiple subsystems with different combinations of $n-1$ species that are unstable. For example, consider a three-species system with species $x_{1}, x_{2}$, and $x_{3}$. Species $x_{1}$ and $x_{2}$ exhibit hydra effects if the $x_{2}, x_{3}$-subsystem is unstable when $x_{1}$ is fixed at $x_{1}^{*}$ and the $x_{2}, x_{3}$-subsystem is unstable when $x_{2}$ is fixed at $x_{2}^{*}$. Second, hydra effects cannot occur for all species at a stable equilibrium; see Appendix S1 for details. Hence, at most $n-1$ species can exhibit hydra effects in a stable $n$-species system; e.g., at most two species in the three species example from above.

\section{APPLICATION OF THE "SUBSYSTEM INSTABILITY" RESUlT TO TWO AND THREE-SPECIES COMMUNITY MODELS}

To demonstrate the utility of condition (2) for identifying species in food webs that may exhibit hydra effects, we apply it to models of stable food webs with two or three species. The small number of species makes it easy to determine the stability of subsystems that lack dynamics of one of those species. The mechanisms that lead to hydra effects for each system are summarized in Table 1. The mathematical details supporting our results are presented in Appendices S3 and S4. Additional examples, including those where hydra effects arise in multiple species in a single web, are also presented in Appendices S3 and S4.

TABLE 1. Mechanisms driving hydra effects in stable two- and three-species communities

\begin{tabular}{|c|c|c|}
\hline System & HE species & Mechanism \\
\hline \multicolumn{3}{|l|}{ Two-species systems } \\
\hline Competition $(-,-)$ & Not possible & \\
\hline Mutualism $(+,+)$ & Not possible & \\
\hline Contramensalism $*(+,-)$ & Species $1 \dagger$ & Positive self-effects for species 2 ; no positive self-effects for species 1 \\
\hline \multirow[t]{2}{*}{ Predator-prey } & Prey & Positive predator self-effects; intraspecific prey competition \\
\hline & Predator & Positive prey self-effects; direct intraspecific predator competition \\
\hline \multicolumn{3}{|l|}{ Three-species systems } \\
\hline Competition & Species $1 \dagger$ & $\begin{array}{l}\text { Species } 2 \text { and } 3 \text { interspecific competition greater than intraspecific competition } \\
\text { or species } 2 \text { competitively excludes species } 3\end{array}$ \\
\hline Mutualism & Not possible & \\
\hline Contramensalism & Species $1 \uparrow$ & $\begin{array}{l}\text { Species } 2 \text { or } 3 \text { has positive self-effects; interspecific interactions between species } \\
2 \text { \& } 3 \text { are weaker than their intraspecific interactions }\end{array}$ \\
\hline \multirow[t]{2}{*}{ Predator-2-prey } & Prey species $1 \uparrow$ & $\begin{array}{l}\text { Positive predator self-effects or increased density of prey } 2 \text { reduces predator } \\
\text { growth rate }\end{array}$ \\
\hline & Predator & $\begin{array}{l}\text { Prey satisfy either condition from 3-species competition system } \\
\text { or prey mutualistic interactions greater than intraspecific interactions } \\
\text { or prey have a contramensalistic interaction and satisfy conditions from } \\
\text { 3-species contramensalistic system }\end{array}$ \\
\hline \multirow[t]{3}{*}{ Food chain } & Bottom species & Middle species has positive self-effects \\
\hline & Middle species & Top or bottom species has positive self-effects \\
\hline & Top species & Middle species has positive self-effects \\
\hline
\end{tabular}

$\dagger$ Due to symmetry, the conditions for hydra effects in the other species are the same after exchanging species labels (e.g., replace species 1 with species 2 and vice versa).

\$Contramensalistic systems with a single trophic level. 
One condition for hydra effects that frequently arises is that one of the species without a hydra effect has "positive self-effects" at the equilibrium. Here, we use positive self-effects to mean that there is a positive relationship between the population growth of a species and its density. We define a positive self-effect as $\partial / \partial x_{j}(\mathrm{~d} x / \mathrm{d} t)>0$ when evaluated at equilibrium. In a closed system (i.e., without immigration), a positive self-effect occurs at equilibrium if and only if there is a positive per capita selfeffect, i.e., $\partial / \partial x_{j}\left(\left(1 / x_{j}\right)(\mathrm{d} x / \mathrm{d} t)\right)>0$, which we refer to as positive density dependence. Positive density dependence can be due to purely intraspecific interactions (e.g., Allee effects) or due to interspecific interactions (e.g., a saturating functional response in a predator of that species).

\section{Hydra effects in two-species communities}

We begin with two-species communities, i.e., $n=2$ in Eq. 1. We first present the general conditions for a species to exhibit a hydra effect. We then discuss particular mechanisms for two-species systems with one trophic level and predator-prey systems.

Species $1\left(x_{1}\right)$ exhibits a hydra effect at a stable equilibrium when three conditions are met. First, species 2 $\left(x_{2}\right.$; the non-HE species) has positive self-effects. Mathematically, this condition arises because the first principal minor of the Jacobian of a two-species system is the diagonal entry of the Jacobian for the second species, i.e., $M_{11}=J_{22}=\partial / \partial x_{2}\left(\mathrm{~d} x_{2} / \mathrm{d} t\right)$. Second, the HE species has negative self-effects, i.e., $J_{11}=\partial / \partial x_{1}\left(\mathrm{~d} x_{1} /\right.$ $\mathrm{d} t)<0$. If this condition is not satisfied, then the coexistence equilibrium is unstable. The third condition is that the effects of increasing each species density on the other species growth rate have opposite signs, i.e., $J_{12}>0>J_{21}$ or $J_{12}<0<J_{21}$. Such a $(+,-)$ interaction structure is known as contramensalism (Arthur and Mitchell 1989).

For two-species systems with one trophic level, hydra effects cannot arise in stable competitive or mutualistic systems. This is because the $(-,-)$ and $(+,+)$ interaction structures in those systems, respectively, do not satisfy the third condition. Contramensalistic interactions $(+,-)$ arise in systems with one trophic level when, e.g., positive environmental modification by one species outweighs its competitive effect on the other species (Arthur 1986). In such cases, a hydra effect can be exhibited by either species. Two examples of contramensalistic systems with hydra effects are presented in Fig. 1.

The most common type of contramensalistic system is a predator-prey system. For such systems, the predator exhibits a hydra effect when the prey has positive selfeffects at equilibrium. This can occur due to processes exclusive to the prey (e.g., Allee effects due to mate finding) or when the predator has a nonlinear (saturating) functional response. In Lotka-Volterra and other commonly used models that do not include direct intraspecific predator competition, positive prey self-effects often result in cyclic predator-prey dynamics. In order for predator hydra effects to arise in a stable system, direct intraspecific predator competition is also necessary, i.e., $\partial / \partial y((1 / y)(\mathrm{d} y / \mathrm{d} t))<0$, where $y$ is predator density. This occurs when the predator per capita mortality rate increases and/or its functional response decreases with predator density. In Fig. 2A, the predator, which exhibits a hydra effect, has a type-II functional response and a nonlinear mortality rate.

Prey hydra effects occur when the predator has positive self-effects at the equilibrium. Positive predator self-effects can arise via predator-dependent functional responses or density-dependent per capita mortality rates where the predator per capita growth rate is maximized
A

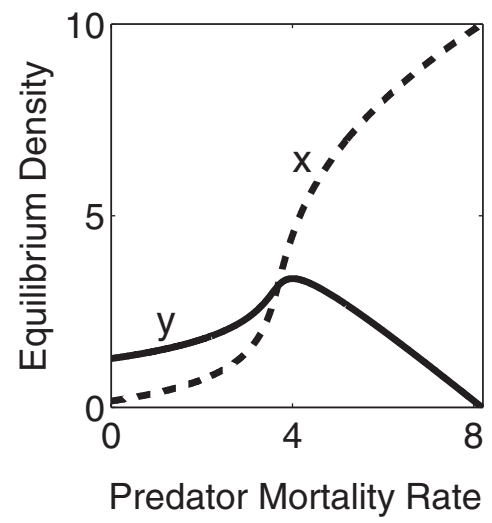

B

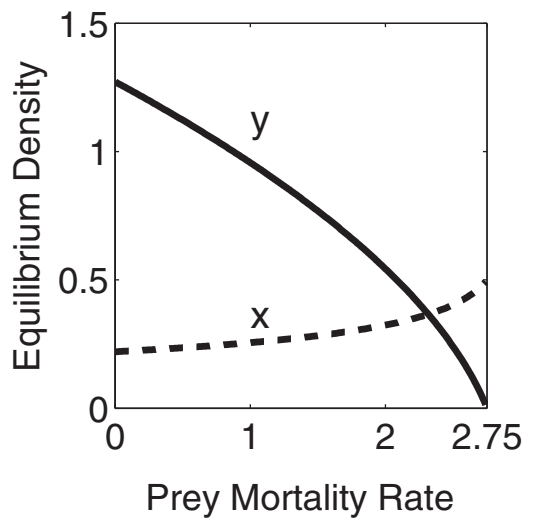

FIG. 1. Examples of hydra effects in two two-species contramensalistic systems. The species having a positive effect on the growth of the other is labeled the cooperator, and the species having a negative effect is called the cheater. Both panels show the equilibrium densities of the cooperator (solid line; $x_{1}$ ) and the cheater (dashed line; $x_{2}$ ) as the mortality rate of (A) the cooperator or (B) the cheater is varied. (A) The cooperator has a hydra effect. Extinction of the cheater $\left(\delta_{1} \approx 0.9\right)$ causes an abrupt change in the response of the cooperator to increases in its own mortality, so that there is no hydra effect at higher mortalities. (B) The cheater has a hydra effect for mortality rates less than $\delta_{2} \approx 0.27$. See Appendix S5 for equations and parameters. 
at intermediate predator densities. Cooperative feeding or hunting among predators can produce such effects. Regardless of the mechanism, the coexistence equilibrium is stable only if the prey per capita growth rate decreases with prey density at the equilibrium, i.e., negative density dependence. Figure $2 \mathrm{~B}$ provides an example where the prey has a hydra effect and the positive predator self-effect is caused by a functional response that depends nonlinearly on predator density.

\section{Hydra effects in models of three species on one trophic level}

We now consider models with three species on the same trophic level. For each pair of species, the interaction can be competition $(-,-)$, mutualism $(+,+)$, or contramensalism $(+,-)$. Examples of models are presented in Appendix S4.

In systems with stable three-species coexistence, one species exhibits a hydra effect if the other two cannot stably coexist when the density of the HE species is fixed at its equilibrium value. This instability of the two-species subsystem can occur via three mechanisms. The first is when the two-species subsystem is bistable because the non-HE species have a competitive or mutualistic interaction and the interspecific effects between them are greater than their intraspecific effects. For a LotkaVolterra system with intraspecific and interspecific competition coefficients $\alpha_{i i}$ and $\alpha_{i j}$, respectively, this would require that $\alpha_{12} \alpha_{21}>\alpha_{11} \alpha_{22}$. For the second mechanism, the two species have a competitive interaction and species one always competitively excludes species two. The third mechanism involves a contramensalistic $(+,-)$ interaction between the two non-hydra species under which one species has positive self-effects, and the product of the interspecific effects between the species are weaker than the product of their intraspecific effects. In all cases, the three species can coexist because the species with the hydra effect suppresses one or both non-HE species.

We highlight when these mechanisms arise in systems with only a single kind of interaction; examples of systems with a mix of interaction types are presented in Appendix S4. Hydra effects cannot arise in stable mutualistic systems. For systems with only competitive interactions, hydra effects can arise (i) via mechanism 2 in rock-paper-scissor-type systems (May and Leonard 1975, Laird and Schamp 2006) where each species excludes and is excluded by one other species or (ii) via mechanism 1 or 2 in systems where the HE species can coexist with either of the other two species in the absence of the third (true for Fig. 5B); an example of each case is given in Fig. 3. For systems with only contramensalistic interactions, hydra effects can only arise via mechanism 3 .

\section{Hydra effects in models with one predator and two prey}

We now consider systems with a generalist predator and two prey, where the prey can have a competitive $(-,-)$, mutualistic $(+,+)$, or contramensalistic $(+,-)$ interaction.

Predator hydra effects occur if stable or neutral coexistence of the two prey is not possible when the predator's density is fixed at its equilibrium value. Predator hydra effects arise via the three mechanisms from the three-species model with a single trophic level: (i) the two-prey subsystem is bistable due to a competitive or mutualistic interaction where interspecific effects are stronger than intraspecific effects; (ii) the prey have a competitive interaction and prey one always competitively excludes prey two; or (iii) the prey have a contramensalistic interaction, one prey has positive self-effects at the equilibrium, and the interspecific effects between the prey are weaker than their intraspecific effects. In all cases, three-species coexistence is possible because the predator suppresses one
A

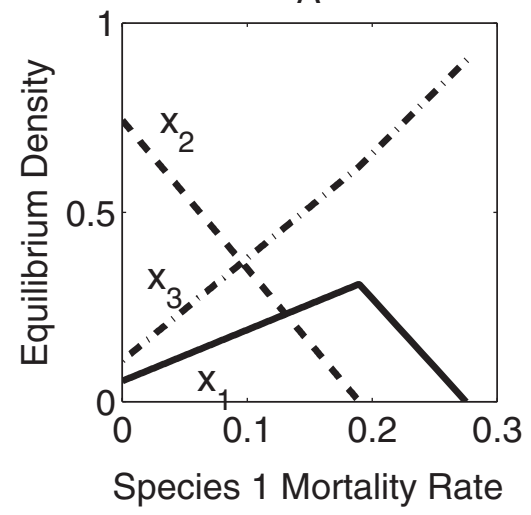

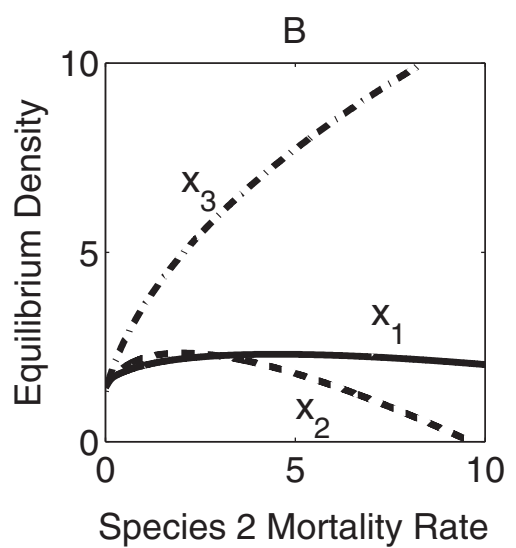

FIG. 2. Examples of predator-prey systems where (A) the predator and (B) the prey has a hydra effect. Both panels show the equilibrium densities of the predator (solid line; $y$ ) and the prey (dashed line; $x$ ) as the mortality rate of (A) the predator or (B) the prey is varied. In (A) the predator only exhibits a hydra effect for mortality rates less than $\delta_{2} \approx 4$. In (B), the prey exhibits a hydra effect for all mortality rates for which the predator exists $\left(\delta_{1}<2.75\right)$. See Appendix S5 for equations and parameters. 
A

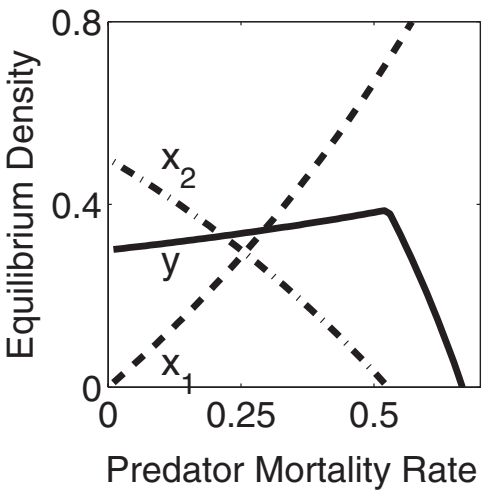

B

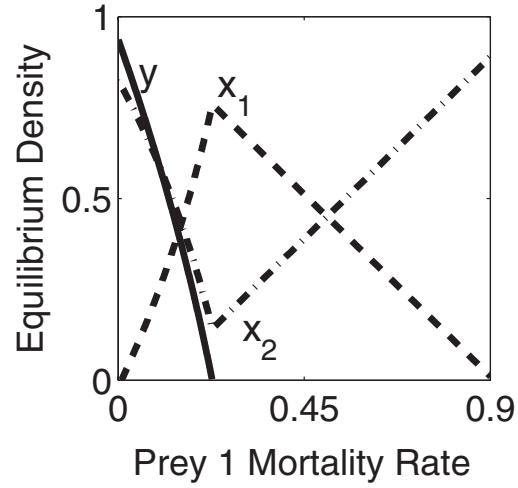

FIG. 3. Examples of hydra effects in systems with three species on the same trophic level. (A) A system with three competitive interactions. (B) A system with three contramensalistic interactions. All panels show the equilibrium densities of species 1 (solid line; $x_{1}$ ), species 2 (dashed line; $x_{2}$ ) and species 3 (dashed-dot line; $x_{3}$ ) as the mortality rate of one species is varied. In (A), extinction of species $2\left(\delta_{1} \approx 0.19\right)$ causes the abrupt change in the response of species $x_{1}$ to increased mortality and the disappearance of its hydra effect. In (B), species $x_{2}$ exhibits a hydra effect for $\delta_{2}<2$. See Appendix S5 for equations and parameters.

A

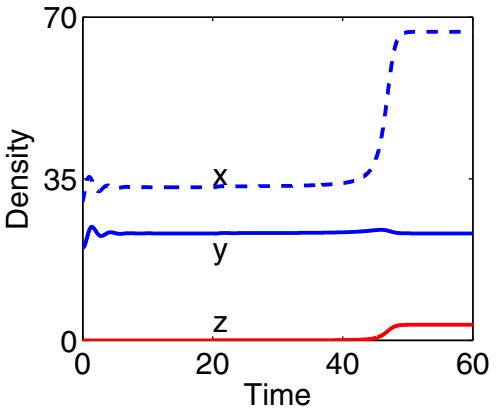

C

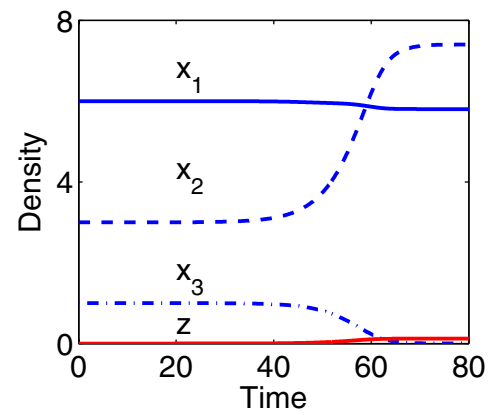

B

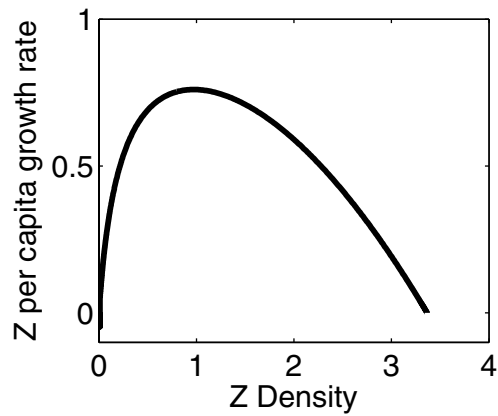

D

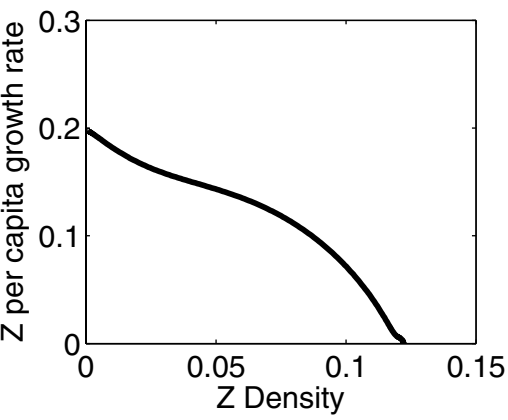

FIG. 4. Introducing a predator that specializes on a species with a hydra effect can cause large changes in community composition, including extinction of one or more species. (A) Predator-prey system (solid blue and dashed blue; $y$ and $x$, respectively) where the predator has a hydra effect. A specialist top predator (red; $z$ ) of the HE predator is introduced at low densities at time $t=20$, which results in large changes in prey density. (C) Three-species competition system where species 1 (solid blue; $x_{1}$ ) has a hydra effect. A specialist predator (solid red; $z$ ) of species 1 is introduced at time $t=20$, which causes the extinction of species 3 (dashed-dot blue; $x_{3}$ ). (B,D) Per capita growth rates of the specialist top predators in $(\mathrm{A})$ and $(\mathrm{C})$, respectively, as functions of their densities. In (B), the specialist top predator has an Allee effect due to the community shift that follows its introduction. See Appendix S5 for equations and parameters.

or both prey. One scenario in which case (ii) is likely to arise is when the predator consumes the competitively superior prey and does not consume the competitively inferior prey; see Fig. 4A, which is based on a model in Abrams and Matsuda (2005). An example of case (iii) is presented in Fig. 4B.
Prey hydra effects occur if the predator cannot coexist with one prey when the density of the other (hydra) prey is fixed at its equilibrium value. Here we focus on the two most biologically likely mechanisms; see Appendix S4 for two others. The first mechanism is that the predator has positive self-effects at the equilibrium. As noted earlier, 
A

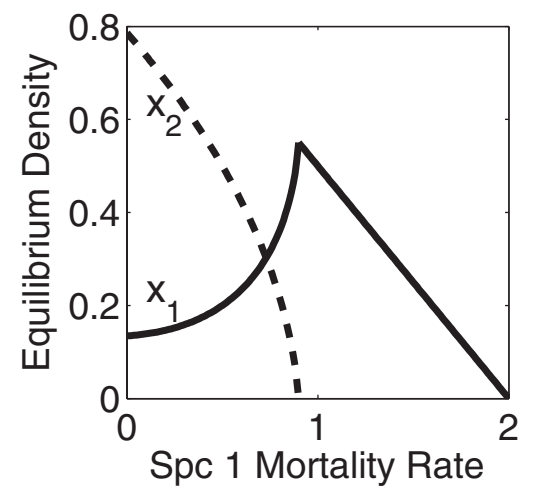

B

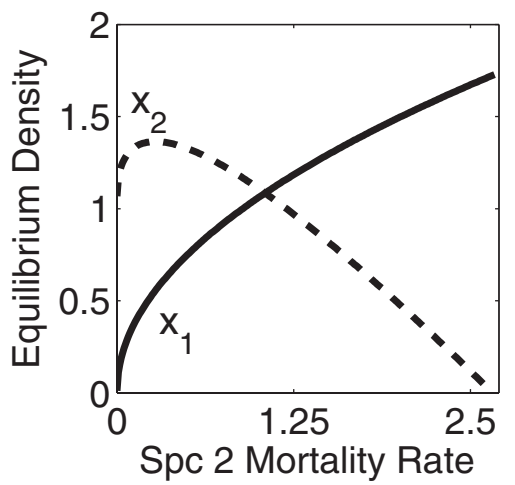

FIG. 5. Examples of one-predator-two-prey systems where the (A) the predator or (B) one prey species has a hydra effect. All panels show the equilibrium densities of the predator (solid line; $y$ ) and the two prey species (dashed and dot-dashed lines; $x_{1}$ and $x_{2}$, respectively) as the mortality rate of (A) the predator or (B) one prey species is varied. In (A), the extinction of prey species 2 $\left(\delta_{3} \approx 0.52\right)$ causes the abrupt change in the response of the predator to increased mortality and the disappearance of its hydra effect. In (B), the abrupt changes in the response of the prey to increased mortality is caused by the extinction of the predator $\left(\delta_{1} \approx 0.23\right)$. See Appendix S5 for equations and parameters.

positive predator self-effects can occur when the predator exhibits cooperative hunting or cooperative defense. Figure $5 \mathrm{C}$ below presents a numerical example of this case. The second mechanism is that increases in the density of the non-hydra prey have a negative effect on the per capita growth rate of the predator. Biologically, this occurs when encounters with the prey species harm the predator (e.g., a chemical or physical defense) or when consuming the prey species causes a net decrease in nutritional intake rate. This requires nonadaptive feeding by the predator, but examples of such behavior do exist (Kratina et al. 2007). With both mechanisms, the predator is often unable to persist when the HE prey is absent from the community.

\section{Hydra effects in simple food chain models}

Finally, we consider a simple three-species food chain model consisting of a basal $(x)$, middle $(y)$, and top $(z)$ species, where the basal and top species do not directly interact. Here we highlight some results about when hydra effects arise and the counterintuitive effects increased mortality of the HE species can have on different trophic levels; see Appendix S4 for more details.

In food chain models, hydra effects are only possible when one species has positive self-effects at equilibrium. In this case, species at adjacent trophic levels can exhibit hydra effects. For example, if the middle species has positive self-effects at equilibrium, then the top or the bottom species can exhibit a hydra effect. Abrams and Vos (2003) found hydra effects in a similar food chain model that did not directly incorporate positive self effects, but did have adaptive foraging by the middle species. While such adaptive change has a number of effects that are not considered here, reduced foraging at higher food densities by the middle species often produces a positive self-effect in the bottom species.
Hydra effects also alter predictions about how indirect effects propagate through the food chain. In the absence of hydra effects, the indirect effects of increased mortality can be predicted by either changes in the mortality rate or changes in the density of the perturbed species. However, if the perturbed species has a hydra effect, then the indirect effects of increased mortality on other species can only be predicted by either the mortality rate or density of the perturbed species, not both; see Appendix S4 for details. Furthermore, in some cases, increased mortality of an HE species can cause all species to increase in abundance. For instance, if the bottom species has positive self-effects at equilibrium, then the middle species always exhibits a hydra effect and increases in the mortality rate of the middle species cause all species to increase in abundance; see example S4-19 of Appendix S4. Longer food chains and more complex food webs also exhibit a much wider range of effect propagation patterns when one or more species exhibit hydra effects.

\section{IMPLICATIONS FOR INVASION BY A PREDATOR OF THE HYDRA-EFFECT SPECIES}

Our results have important implications for understanding the impacts of predator addition on system stability. Introducing a specialist, food-limited predator that consumes an HE species will constrain the dynamics of the HE species and consequently, destabilize the system under the same circumstances that fixing the HE species' density does so. This is shown in Appendix S2. (The introduced predator may destabilize the dynamics under additional circumstances if it has a saturating functional response, but we ignore that possibility here.) Large changes in densities, including extinction, may occur as a result of the destabilization, even when the change in the abundance of the HE species is minimal. Other 
sources of density-dependent mortality can produce similar community shifts; this includes harvesting with feedback control and adaptively switching generalist predators.

Figure 5 presents two examples of newly introduced specialist predators causing community shifts in a predator-prey system (Fig. 5A) and a three-species competition community (Fig. 5C). In both panels, the solid blue species exhibits a hydra effect. Introducing a specialist predator of that species (solid red) at low densities destabilizes the system, eventually causing large changes in the community makeup. In Fig. 5C, one of the non-hydra species (dash-dot blue) is driven to extinction. In Fig. 5A, initially the specialist can only persist at low densities. However, the shift in the community makeup following its introduction allows the specialist predator to reach much higher densities (Fig. 5B). Hence, a hydra effect in one species can produce an emergent Allee effect (de Roos et al. 2003) in its specialist predator; the specialist predator alters its environment and causes community shifts that result in better conditions for its own growth, which allows it to reach higher population densities. In some cases this emergent Allee effect may be strong. Note that hydra effects do not always produce an emergent Allee effect for an introduced specialist predator (Fig. 5D).

Our results also show how hydra effects alter the community impacts of predator addition. First, different sources of mortality can have different effects on the density of HE species. Because it is an added source of mortality for the HE species, one might expect an introduced specialist predator to cause the HE species' density to increase. However, increases in the HE species' density cause an increase in the specialist predator's density, which in turn increases the HE species' mortality further. Such a positive feedback must end at some point, which means that the specialist predator's equilibrium density must become high enough to eliminate the hydra effect in its prey at the new system equilibrium. The net result is that the introduced specialist causes the HE species' density to decrease.

Second, indirect effects caused by an introduced predator of an HE species can be amplified as they propagate through a food web. For example, introduction of the specialist predator $(z)$ in Fig. 5A causes a small decrease in the density of the HE species $(y)$ and a large increase in the density of the prey $(x)$ of the HE species. Since the introduced specialist predator occupies the top trophic level, this scenario represents a normal trophic cascade, except that the proportional change in prey density (bottom trophic level) is much larger than the proportional change in the density of the HE species (middle trophic level). This means the indirect effect of the specialist predator on the prey of the HE species is larger than its direct effect on the HE species. Amplification of an indirect effect can also occur when the introduced predator of the HE species does not occupy the top trophic level. This is significant because Schoener (1993) argued, even slow dampening of indirect effects makes prediction of population changes very difficult in community ecology.

Third, indirect effects caused by an introduced predator of an HE species can have counterintuitive sign structures as they propagate through a food web. For example, if the density of an introduced generalist predator of an HE species is controlled by other factors, then the additional mortality experienced by the HE species is a fixed increase in its per capita mortality rate. For the predator-prey system in Fig. 2A where the predator species $y$ exhibits a hydra effect, an increase in such a generalist predator's density would cause an increase in the per capita mortality rate of predator $y$ and, counterintuitively, cause both the mid and bottom trophic levels to increase. The underlying mechanism driving this behavior is that in order for $y$ to increase in abundance with greater mortality, the density of the bottom prey $(x)$ must increase to balance the increased mortality of species $y$.

\section{Discussion}

Our general result reveals that hydra effects occur in a much wider range of systems than had previously been appreciated and that, in those systems, hydra effects are characterized by instability of the rest of the community when the hydra effect species is maintained at a fixed density. These results have important implications for conservation biology and management strategies of exploited populations, as well as for community ecology generally. Because hydra effects involve increased abundance following increased mortality and decreased abundance following increased immigration, changes in the population size of an $\mathrm{HE}$ species in response to environmental change often have the opposite meaning of similar changes in non-hydra species. Thus, if the goal of management is to increase the density of a species to a particular population level, then, counterintuitively, increased, rather than decreased, mortality of an $\mathrm{HE}$ species is needed. This is complicated by the fact that the initial, transient response of an $\mathrm{HE}$ species to altered mortality is usually (Abrams 2002) in the opposite direction of the final response, so management changes would have to take this time lag into account. As noted in Abrams (2002), population increases due to the hydra effect indicate declines rather than increases in environmental quality, as reflected in maximum individual fitness or per capita growth rate. Our results also show that in many cases $\mathrm{HE}$ species are important for maintaining community structure and diversity. Either the loss of a species with a hydra effect or the introduction of top-down control via a predator that specializes on the HE species can cause large community changes, and often drive other species to extinction. The example in Fig. $5 \mathrm{~A}$ is a classic case where the hydra-species is a keystone predator, allowing coexistence of its two prey. An introduced specialist predator of that keystone species, even if it produces a minimal change in the 
keystone species' density, can destroy prey coexistence. While we have focused on specialist predators, introduced generalist predators are also likely to cause community shifts, provided the generalists have sufficiently weak interactions with non-hydra species. Importantly, destabilization can also occur when the top-down control is a management strategy where the density of the HE species is maintained at a target level (e.g. an optimal yield density in fisheries). Thus, it is important to determine whether a species can exhibit a hydra effect before adopting such a management strategy.

Hydra effect species frequently affect the nature of trophic cascades. Hydra effect species can amplify indirect effects as they propagate through communities. For example, in Fig. 5A, the introduced specialist predator $(z)$ has a small effect on the density of the HE predator species $(y)$ and much larger effects on the density of its prey $(x)$. This example contradicts standard theory about trophic cascades, where the strength of the cascade is often defined (e.g., Heath et al. 2014, p. 101) as "the extent to which a disturbance is diminished as it propagates through a food web." Hydra effects also affect predictions about the potential role of density dependent mortality in dampening indirect effects in interaction-webs (Schoener 1993). External mortality applied to an HE species that experiences density dependent mortality will have a larger effect on its population than in the absence of such density dependent mortality. This has the potential to magnify the indirect effects produced by that external mortality on other species that interact with the HE species. Hydra effects can also change the sign of indirect effects and yield counterintuitive responses to increased mortality in the HE species. For example, in three-species food chain models where the bottom species has an Allee effect, increased mortality of the middle species results in increased density at all trophic levels.

Many of the circumstances we identified as producing hydra effects have not previously been recognized. For example, Gilpin (1975) illustrated cycles in predator-prey systems with positive prey self-effects due to both prey Allee effects and a type-II predator functional responses without noting the positive effect of mortality on average population size. Gilpin's (1979) analysis of chaotic dynamics ("spiral chaos") in a model of one predator and two competing prey did not note the hydra effect that characterized the predator in the model. Early studies of 3-species Lotka-Volterra competition models (Strobeck 1973, May and Leonard 1975) discussed examples which exhibited hydra effects without recognizing them. In addition, our results show that there are numerous classes of counter-examples to a recent assertion that hydra effects in predator-prey systems only occur in predators and require type-II predator functional responses, logistic resource growth and population cycles (Schröder et al. 2014, 2015). Our results also help understand and generalize the findings of Frean and Abraham (2001), who found that the weakest competitor had the largest population size in models of intransitive (rockpaper-scissors) competition.

There are many more three-species models that could be considered (e.g., plant-herbivore-pollinator and intraguild predation models), and even more possibilities for models of four or more species. This paper would be far too long if we addressed them all here. However, our general condition (2) applies to communities of any size. Hence, condition (2) can be used to identify the biological circumstances under which hydra effects arise in systems beyond those considered here. Moreover, the many circumstances leading to hydra effects in the twoand three species webs discussed above suggests that hydra effects are likely in larger interaction-webs. Abrams and Cortez (2015) showed that hydra effects were common for consumer species in a 2-consumer2-resource Lotka-Volterra system having asymmetrical competition between resources. Exploration of larger webs will no doubt uncover many unappreciated mechanisms for hydra effects. Previous results imply such effects in large food webs even though they were not described as such. Yodzis (1988) observed cases where species had decreased density in response to increased immigration in $27 \%$ of the 223 species present in the 16 empirically based Lotka-Volterra models of food webs he analyzed. Koen-Alonso and Yodzis (2005) subsequently found hydra effects for two of the species in a more detailed four-species model of a Patagonian marine community; again these were only identified as negative self-effects. Wollrab et al. (2012) found that hydra effects were possible for top predators in 5 of 19 food webs consisting of two linked food chains, provided that top-predator functional responses were saturating. Again, these were not identified as hydra effects. The fact that hydra effects arise in so many community models when the authors are not looking for them argues that they are also likely to occur frequently in natural systems.

It is unlikely that hydra effects occur nearly as often as the traditional negative response of population size to increased mortality. However, there is limited evidence available for judging the frequency of these effects in natural communities. The introduction lists studies that have experimentally documented hydra effects, and several less conclusive studies are mentioned in Abrams $(2009>b)$ and Schröder et al. (2014). This scarcity of examples is not surprising given the limited time since recent theory first revived Ricker's (1954) idea that populations might increase with greater mortality. The wide range of community models where hydra effects have been demonstrated also argues that many more such effects exist, both in nature and in models. Many of the biological conditions for hydra effects in the two- and three-species models we considered are expected to be common in natural systems. These conditions require positive self-effects in some species and negative selfeffects in others. Negative self-effects are extremely common, even in consumer species (DeLong and Vasseur 
2011, Heath et al. 2014). Causes of positive self-effects that are known to be common include type-II functional responses (Jeschke et al. 2004) and Allee effects (Kramer et al. 2009).

While our general result does apply to a large class of systems, there are several mechanisms for hydra effects that cannot be described using our approach. Abrams $(2009>b)$ reviewed the following mechanisms: (1) altered cycle amplitude with increasing mortality in unstable systems; (2) mortality factors acting on predators that have the side effect of decreasing their per capita consumption rate of prey; (3) overcompensation due to scramble competition in difference equation models and in continuous time models with stage-structured populations. The third mechanism has been explored and discussed extensively for size-structured communities by de Roos and Persson (2013). Our analysis does not apply to difference equation models (e.g., Liz and Pilarczyk 2012), or to differential equation models where cycles play an essential role in producing the hydra effect (e.g., Abrams et al. 2003, Sieber and Hilker 2012). However, it does have implications for other cases. If the dynamics of all size classes are described by ordinary differential equations (as in Abrams and Quince 2005, de Roos et al. 2007 and Schreiber and Rudolf 2008), then the condition for a hydra effect for any particular class in a stable system implies instability of the subsystem in which the abundance of that class is fixed or experiences top-down control (e.g., by a size-specific specialist predator). For systems with dynamic phenotypic traits (e.g., Abrams and Vos 2003, Matsuda and Abrams 2004, Abrams and Matsuda 2005, Abrams 2012), a hydra effect implies instability in the trait-population subsystem in which the density of the hydra effect species is fixed. This differs from the models considered above only in that the instability caused by fixing the density of the HE species may lead to large evolutionary changes as well as or instead of population changes. Abrams (2012) provides an example of such a shift caused by introducing a specialist predator into a generalist-predator-2-prey system with evolution in the resident predator and a hydra effect in both prey. Some cases with hydra effects driven by adaptive change in prey traits have the same properties as models having additional species in which the mean trait of a trophic level changes based on shifts in species abundances (Abrams and Matsuda 2005).

Many previous examples of hydra effects have exhibited cyclic (Abrams 2002, Abrams et al. 2003, Abrams 2009a,b, Sieber and Hilker 2012, Guill et al. 2014) or chaotic dynamics (Gilpin 1979, Abrams et al. 2003). Current theory and empirical results are insufficient to argue whether hydra effects are more likely in stable or unstable systems. Although we have only considered systems with stable equilibria, our general result only requires that the sign of the determinant of the Jacobian be the same as that for an equilibrium that is stable. Thus, our results about subsystem instability may also apply to systems with fluctuating population densities, provided that the time-averaged and equilibrium densities for a species respond to increased mortality in the same direction. Sieber and Hilker (2012) show that this is always true for at least part of the range of mortalities with instability in nonlinear predator-prey models. Example S4-3 in Appendix S4 shows that a Lotka-Volterra three-species competition model may be stabilized or destabilized without changing the hydra effect. This suggests that maintaining the HE species at a fixed density or otherwise controlling its density (e.g., via a specialist predator) in a cyclic system may cause the extinction of one or more other species. This destabilization has been previously observed in predator-prey models with predator hydra effects where the cyclic dynamics are driven by prey Allee effects or predator type-II functional responses (Matsuda and Abrams 1994).

\section{ACKNOWLEDGMENTS}

PAA was supported by a Discovery Grant from the Natural Sciences and Engineering Research Council of Canada. MHC and PAA designed the study, analyzed the models and wrote the paper.

\section{Literature Cited}

Abrams, P. A. 2002. Will declining population sizes warn us of impending extinctions? American Naturalist 160:293-305.

Abrams, P. A. 2009a. Adaptive changes in prey vulnerability shape the response of predator populations to mortality. Journal of Theoretical Biology 261:294-304.

Abrams, P. A. 2009b. When does greater mortality increase population size? The long history and diverse mechanisms underlying the hydra effect. Ecology Letters 12:462-474.

Abrams, P. A. 2012. The eco-evolutionary responses of a generalist consumer to resource competition. Evolution 66: 3130-3143.

Abrams, P. A., C. E. Brassil, and R. D. Holt. 2003. Dynamics and responses to mortality rates of competing predators undergoing predator-prey cycles. Theoretical Population Biology 64:163-176.

Abrams, P. A., and M. H. Cortez. 2015. The many potential interactions between predators that share competing prey. Ecological Monographs. 85:625-641.

Abrams, P. A., and H. Matsuda. 2005. The effect of adaptive change in prey on the dynamics of an exploited predator population. Canadian Journal of Fisheries and Aquatic Sciences 62:758-766.

Abrams, P. A., and C. Quince. 2005. The impact of mortality on predator population size and stability in systems with stage-structured prey. Theoretical Population Biology 68: 253-266.

Abrams, P. A., and M. Vos. 2003. Adaptation, density dependence and the responses of trophic level abundances to mortality. Evolutionary Ecology Research 5:1113-1132.

Arthur, W. 1986. On the complexity of a simple environment: competition, resource partitioning and facilitation in a two-species Drosophila system. Philosophical Transactions of the Royal Society B 313:471-508.

Arthur, W., and P. Mitchell. 1989. A revised scheme for the classification of population interactions. Oikos 56:141-143.

de Roos, A. M., and L. Persson. 2013. Population and community ecology of ontogenetic development. Princeton University Press, Princeton, NJ. 
de Roos, A. M., L. Persson, and H. R. Thieme. 2003. Emergent Allee effects in top predators feeding on structured prey populations. Proceedings of the Royal Society of London B: Biological Sciences 270:611-618.

de Roos, A. M., T. Schellekens, T. van Kooten, K. van de Wolfshaar, D. Claessen, and L. Persson. 2007. Fooddependent growth leads to overcompensation in stage-specific biomass when mortality increases: the inuence of maturation versus reproduction regulation. American Naturalist 170:E59-E76.

DeLong, J. P., and D. A. Vasseur. 2011. Mutual interference is common and mostly intermediate in magnitude. BMC Ecology 11:1.

Frean, M., and E. R. Abraham. 2001. Rock-scissors-paper and the survival of the weakest. Proceedings of the Royal Society B: Biological Sciences 268:1323-1327.

Fryxell, J. M., I. M. Smith, and D. H. Lynn. 2005. Evaluation of alternate harvesting strategies using experimental microcosms. Oikos 111:143-149.

Georgelin, E., and N. Loeuille. 2014. Dynamics of coupled mutualistic and antagonistic interactions, and their implications for ecosystem management. Journal of Theoretical Biology 346:67-74.

Gilpin, M. E. 1975. Group selection in predator-prey communities. Princeton University Press. Princeton, New Jersey, USA.

Gilpin, M. E. 1979. Spiral Chaos in a predator-prey model. The American Naturalist 113:306-308.

Guill, C., E. Carmack, and B. Drossel. 2014. Exploring cyclic dominance of sockeye salmon with a predator-prey model. Canadian Journal of Fisheries and Aquatic Sciences 71: 959-972.

Heath, M. R., D. C. Speirs, and J. H. Steele. 2014. Understanding patterns and processes in models of trophic cascades. Ecology Letters 17:101-114.

Jeschke, J. M., M. Kopp, and R. Tollrian. 2004. Consumer-food systems: why type I functional responses are exclusive to filter feeders. Biological Reviews 79:337-349.

Koen-Alonso, M., and P. Yodzis. 2005. Multispecies modelling of some components of the marine community of northern and central Patagonia, Argentina. Canadian Journal of Fisheries and Aquatic Sciences 62:1490-1512.

Kramer, A. M., B. Dennis, A. M. Liebhold, and J. M. Drake. 2009. The evidence for Allee effects. Population Ecology 51: 341-354.

Kratina, P., M. Vos, and B. R. Anholt. 2007. Species diversity modulates predation. Ecology 88:1917-1923.

Laird, R. A., and B. S. Schamp. 2006. Competitive intransitivity promotes species coexistence. American Naturalist 168: 182-193.

Liz, E., and P. Pilarczyk. 2012. Global dynamics in a stagestructured discrete-time population model with harvesting. Journal of Theoretical Biology 297:148-165.
Matsuda, H., and P. A. Abrams 1994. Timid consumers: selfextinction due to adaptive change in foraging and anti-predator effort. Theoretical Population Biology 45:76-91.

Matsuda, H., and P. A. Abrams 2004. Effects of predator-prey interactions change on sustainable yield. Canadian Journal of Fisheries and Aquatic Sciences 61:175-184.

May, R. M., and W. J. Leonard 1975. Aspects of competition between three species. SIAM Journal on Applied Mathematics 29:243-253.

Nicholson, A. J. 1954. Compensatory reactions of populations to stresses, and their evolutionary significance. Australian Journal of Zoology 2:1-8.

Nicholson, A. J. 1957. The self-adjustment of populations to change. Pages 153-173 in Cold spring harbor symposia on quantitative biology. Cold Spring Harbor Symposia on Quantitative Biology 22:153-173.

Ricker, W. E. 1954. Stock and recruitment. Journal of Fisheries Research Board of Canada 11:559-623.

Schoener, T. W. 1993. On the relative importance of direct versus indirect effects in ecological communities. Pages 365-411 in H. Kawanabe, J. E. Cohen, and K. Iwasaki, editors. Mutualism and community organization: behavioral, theoretical, and foodweb approaches. Oxford University Press, New York, NY.

Schreiber, S., and V. H. W. Rudolf 2008. Crossing habitat boundaries: coupling dynamics of ecosystems through complex life cycles. Ecology Letters 11:576-587.

Schröder, A., L. Persson, and A. M. de Roos 2009. Culling experiments demonstrate size-class specific biomass increases with mortality. Trends in Ecology and Evolution 29:614-624.

Schröder, A., van Leeuwen, and T. C. Cameron 2014. When less is more: positive population-level effects of mortality. Trends in Ecology and Evolution 29:614-624.

Schröder, A., A. van Leeuwen, and T. C. Cameron. 2015. Empirical support for different types of positive mortality effects. A reply to Abrams. Trends in Ecology and Evolution in press. 30:180-181.

Sieber, M., and F. M. Hilker 2012. The hydra effect in predator-prey models. Journal of Mathematical Biology 64:341-360.

Sieber, M., H. Malchow, and F. M. Hilker 2014. Diseaseinduced modification of prey competition in eco-epidemiological models. Ecological Complexity 18:74-82.

Strobeck, C. 1973. N species competition. Ecology 54:650-654.

Wollrab, S., S. Diehl, and A. M. de Roos. 2012. Simple rules describe bottom-up and top-down control in food webs with alternative energy pathways. Ecology Letters 15:935-946.

Yodzis, P. 1988. The indeterminacy of ecological interactions as perceived through perturbation experiments. Ecology 69: 508-515.

Zipkin, E. F., P. J. Sullivan, E. G. Cooch, C. E. Kraft, B. J. Shuter, and B. C. Weidel. 2008. Overcompensatory response of a smallmouth bass (Micropterus dolomieu) population to harvest: release from competition? Canadian Journal of Fisheries and Aquatic Sciences 65:2279-2292.

\section{SUPPORTING INFORMATION}

Additional supporting information may be found in the online version of this article at http://onlinelibrary.wiley.com/ doi/10.1890/15-0648.1/suppinfo 